\title{
Protótipo de Alta Fidelidade de Assistente Virtual para Instituições de Ensino no Estado do Amazonas
}

\author{
Ana Paula Cardoso Teixeira \\ Instituto Federal do Amazonas - IFAM \\ Manaus, AM, Brasil \\ 2020003166@ifam.edu.br \\ Paulo Victor Marajó \\ Instituto Federal do Amazonas - IFAM \\ Manaus, AM, Brasil \\ 2020008402@ifam.edu.br
}

\author{
Yuri Farias dos Santos \\ Instituto Federal do Amazonas - IFAM \\ Manaus, AM, Brasil \\ 2017004949@ifam.edu.br \\ Priscila Silva Fernandes \\ Instituto Federal do Amazonas - IFAM \\ Manaus, AM, Brasil \\ priscila.fernandes@ifam.edu.br
}

\author{
Edimar Rodrigues Junior \\ Instituto Federal do Amazonas - IFAM \\ Manaus, AM, Brasil \\ 2018004569@ifam.edu.br
}

\author{
Vitor Bremgartner da Frota \\ Instituto Federal do Amazonas - IFAM \\ Manaus, AM, Brasil \\ vitorbref@ifam.edu.br
}

\begin{abstract}
In educational institutions, the use of virtual assistants has been encouraged due to the possibility of disseminating relevant information, assisting in communication between actors and providing support in organizing academic activities. Despite the existence of several virtual assistants, these systems do not present real-time information or data at a personalized level of detail. In addition, the systems that provide this information are not free of charge, making it difficult for institutions with low resources to adopt them. Considering the importance of assisting students and teachers during academic activities through the provision of information, this work presents a proposal for a virtual assistant application for Federal Institute of Amazonas Campus Manaus Distrito Industrial (IFAM/CMDI). Brainstorming and benchmarking techniques were applied to survey the functionalities to be presented by the system and a high-fidelity prototype of the system was developed. As a result, it was possible to present the various dialogues that the assistant will provide and the types of information that will be presented to the various actors within the educational institution.
\end{abstract}

\section{PALAVRAS-CHAVE}

Engenharia de Software, Sistemas de Informação, Interação HumanoComputador

\section{INTRODUÇÃO}

No decorrer da vida acadêmica de um aluno do ensino superior para o mesmo conseguir informações vem a se utilizar de várias ferramentas para auxiliar na organização de sua rotina na instituição. Os professores e corpo acadêmico, no que lhes concerne, são responsáveis pelo fornecimento dessas informações. No entanto, essa comunicação, por vezes, acaba não chegando para todos os alunos da mesma forma. Mesmo com portais institucionais, o tempo para atualização de comunicados é demorado e muitas não incluem informações que parecem simples, mas que são de grande importância para os estudantes naquele momento.
Segundo Meşe e Sancak [1], as ferramentas utilizadas nos ambientes educacionais, bem como aquelas utilizadas pelos alunos, são diversificadas em função da mudança de tecnologia. O uso de assistentes virtuais tem sido encorajado devido à possibilidade de disseminar informações relevantes, auxiliar na comunicação entre os atores e fornecer suporte na organização de atividades acadêmicas. Para Kulothunkan et al. [2], o chatbot para educação tem grande potencial para complementar educadores humanos e administradores de educação. Reshmi e Balakrishnan [3] afirmam que o chatbot é um software que responde à entrada de linguagem natural e tenta manter uma conversa de uma forma que imita uma pessoa real.

Apesar da existência de diversos assistentes virtuais, estes sistemas não apresentam informações em tempo real ou dados a um nível de detalhamento personalizado. Além disso, os sistemas que fornecem estas informações não têm custo gratuito, dificultando sua adoção por instituições com poucos recursos. Nesse contexto, este artigo apresenta uma proposta de sistema que atende às necessidades descritas acima.

\section{O SISTEMA}

\subsection{Metodologia de Concepção da Proposta}

Para que fosse possível o levantamento de dados usamos o framework SCRUM. Para cada "sprint" foram levantadas informações para definir quais seriam as técnicas de elicitação de requisitos que mais se encaixavam para o projeto. Costa et al. [4] afirmam que a atividade de elicitação executada desde o início do projeto tem um grande impacto nas atividades subsequentes e é essencialmente humana e subjetiva.

Considerando que os autores deste trabalho fazem parte do público alvo da instituição onde será implantado o sistema, foi utilizada a técnica de grupo de foco. Nesta técnica foi usada a comunicação verbal, e os participantes puderam expressar suas necessidades e com isso foi possível obter informação qualitativa para a pesquisa. Para Silva et al. [5], a engenharia de requisitos tem buscado, por meio de técnicas de criatividade, fornecer meios para elicitação de 
requisitos úteis e inovadores. Com o levantamento feito durante o grupo de foco, foi possível utilizar a técnica de Brainstorming, gerando assim as características e requerimentos da solução proposta. Isso aliado a uma pesquisa por sistemas com funcionalidades similares às propostas pela solução desejada. A Tabela 1 mostra os resultados obtidos.

\subsection{O Protótipo Desenvolvido}

Com os dados levantados foi possível gerar o diagrama de atividades mostrado na Figura 1, detalhando o funcionamento proposto para o sistema. Diante disso, nossa proposta é um sistema que reúne todas as informações relevantes para melhor auxiliar na comunicação entre alunos e instituição.

Esse aplicativo serve para auxiliar os alunos do Instituto Federal do Amazonas Campus Manaus Distrito Industrial (IFAM/CMDI) para obter informações de baixa e média complexidade, que levariam algum tempo para serem adquiridas por vias comuns. Considerando os resultados obtidos, foi gerado um protótipo de alta fidelidade usando a plataforma web Figma (editor gráfico de prototipagem, adaptável aos principais sistemas operacionais, além de possuir uma versão gratuita), a fim de simular as funcionalidades propostas pelo nosso sistema.

O sistema realiza uma interação com o usuário através de um chatbot, respondendo as perguntas do mesmo, como localização de salas de aula, disponibilidade de livros, horários, notícias e comunicados da instituição para os alunos. A Figura 2 mostra o protótipo do aplicativo.

\section{CONSIDERAÇÕES FINAIS}

Considerando os resultados obtidos, esse aplicativo serve para auxiliar os alunos de uma instituição em obter informações de baixa e média complexibilidade, que levariam algum tempo para serem adquiridas por vias normais. Com isso, foi possível analisar que aplicativos desse gênero além de viável atualmente, podem proporcionar resultados satisfatórios na resolução de dúvidas simples, e em pequenos problemas de qualidade de vida acadêmica.

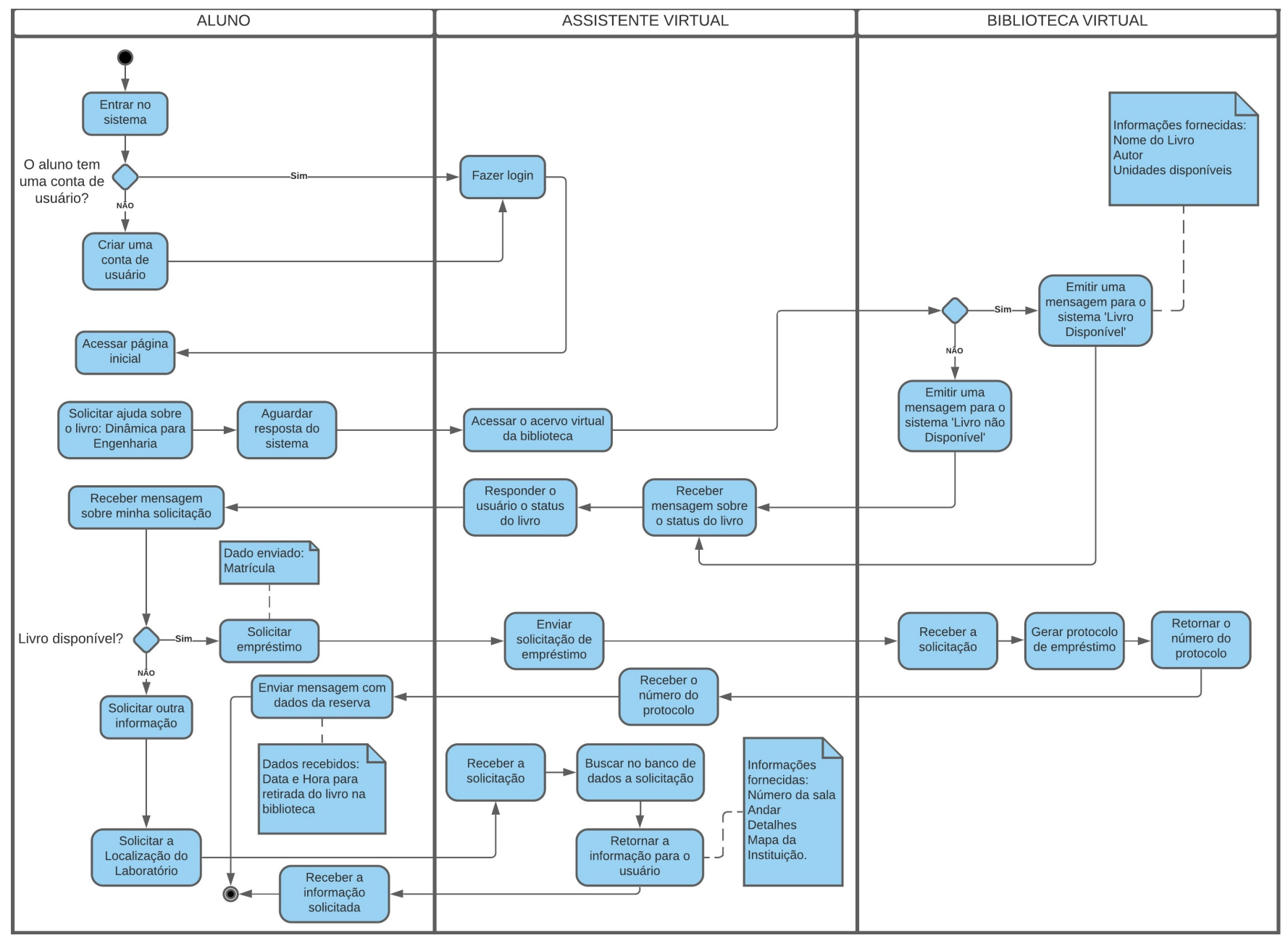

Figura 1: Diagrama de atividades do sistema. 
Tabela 1: Listagem de problemas levantados pelo grupo de foco e suas soluções planejadas via brainstorming.

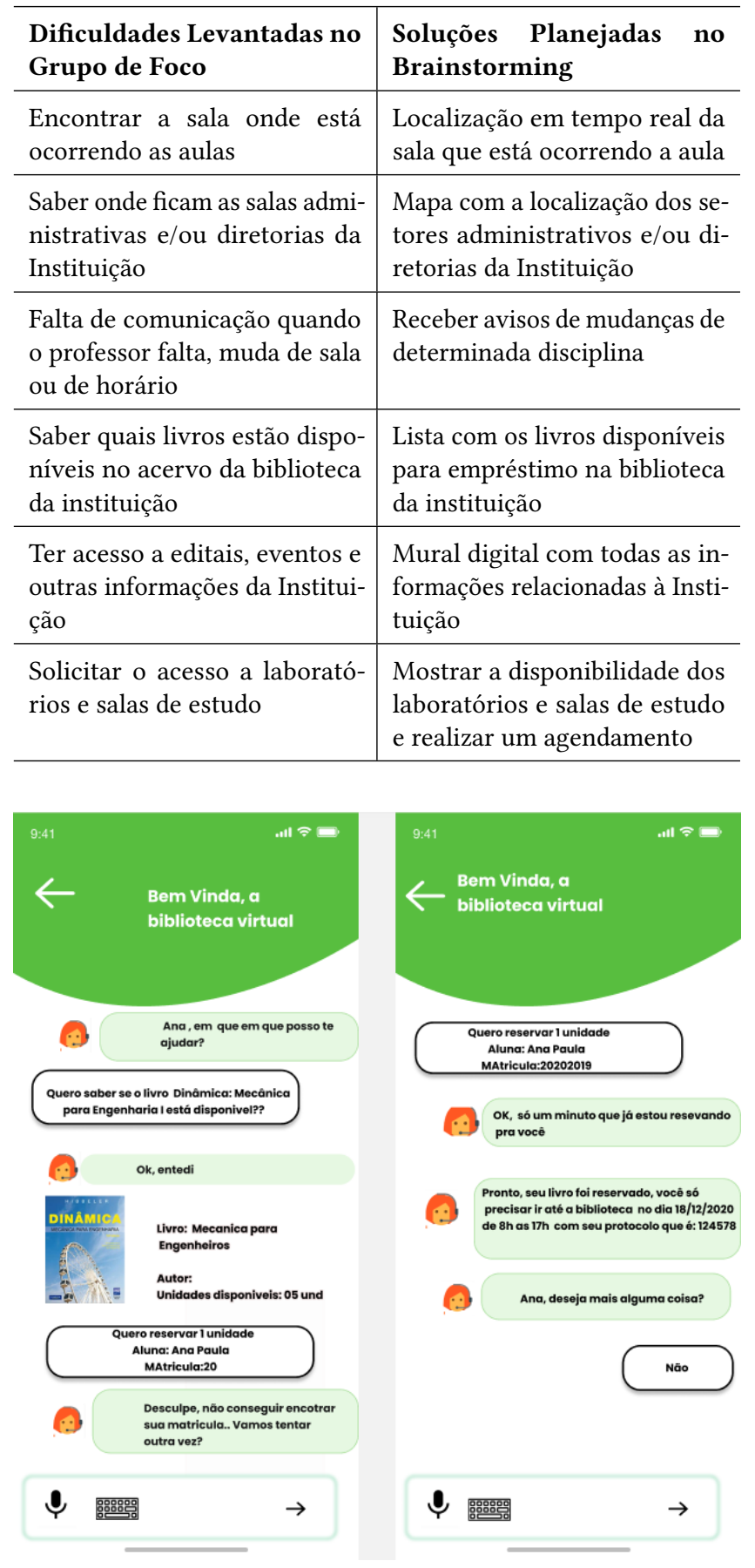

Figura 2: Telas do Protótipo de Alta Fidelidade do Sistema.

O aplicativo, embora ainda não implementado, pode vir a ser ampliado em suas funcionalidades, tornando-o mais atrativo a uma eventual implementação em instituições de ensino, provando-se uma alternativa para a facilitação da comunicação entre as instituições e seus alunos.

\section{AGRADECIMENTOS}

Este trabalho foi realizado com o apoio do Projeto Aranouá, convênio realizado entre o Instituto Federal do Amazonas (IFAM) e a Samsung Eletrônica da Amazônia (SEDA).

\section{REFERENNCIAS}

[1] Can Mese and Gökçe Sancak Aydin. The use of social networks among university students. Educational Research and Reviews, 14(6):190-199, 2019.

[2] Kulothunkan Palasundram, Nurfadhlina Mohd Sharef, Nurul Nasharuddin, Khairul Kasmiran, and Azreen Azman. Sequence to sequence model performance for education chatbot. International fournal of Emerging Technologies in Learning (ifET), 14(24):56-68, 2019.

[3] S Reshmi and Kannan Balakrishnan. Implementation of an inquisitive chatbot for database supported knowledge bases. sādhanā, 41(10):1173-1178, 2016.

[4] La Costa and Alessandra Zoucas. Elicitação de requisitos de software no setor público: lições aprendidas e recomendações para mitigação de riscos. Revista Brasileira de Administração Científica, 3(2):214-226, 2012.

[5] Lyrene Fernandes Silva, Rafael Pinto, and Márcia Lucena. Analisando uma técnica de elicitação baseada na criatividade combinacional-um estudo comparativo. Revista Brasileira de Computação Aplicada, 9(4):105-119, 2017. 